\title{
Economic Design of the Special VSSI T² Chart with Genetic Algorithms Based on Markov Chain
}

\author{
Li-Jun GU ${ }^{1, a,{ }^{*}}$, Qing-Guo TANG ${ }^{1, b}$
}

${ }^{1}$ School of Economics and Management, Nanjing University of Science and Technology , Nanjing 210094, China

agulijunsho@outlook.com, btangqguo@163.com

Keywords: Multivariate control chart, SVSSI, Genetic algorithms, Economic design.

\begin{abstract}
T^{2}$ control chart is widely used to monitor the multivariate process for quality improvement. In this paper, economic performance analysis on the SVSSI $T^{2}$ control chart is studied. Two statuses whether the process is in control or out of control are considered to define the states of Markov Chain. And a numerical example is provided to compare the performances of three control charts in terms of AATS and EL. The results show that the performance of SVSSI control chart is better than other charts. Further, the genetic algorithm is used to search the optimal solution of the SVSSI $T^{2}$ control chart.
\end{abstract}

\section{Introduction}

In an increasingly competitive and diversified era, taking advantage of quality is an important condition for companies to capture the market. As we all know, the control chart is most widely used to detect the occurrence of assignable cause as a powerful quality control tool . In the actual production process, due to the complexity of the product and the diversification of consumer demand, the quality indicators of production are often more than one parameter. In addition to that, they are not independent of each other but mutual influence, which involves a multivariate quality control and also a hot issue in the field of research. Hotelling (1947) proposed the first multivariate control chart, which marked the quality management into the era of multivariate quality control.

There are three main parameters that determine the effect of the control chart: sampling interval, sampling size and control limit. On this basis, Reynoldst (1988) proposed first variable sampling interval mean control chart, which gradually formed a new research field, known as the adaptive quality control chart[1]. There are four types of adaptive control chart: the variable sampling interval(VSI), the variable sampling size(VSS), the variable sampling size and sampling interval(VSSI) and the variable parameter(VP) charts.

SVSSI control chart is a special VSSI chart, which uses two values of the sampling interval and three values of the sampling size. It is proposed by Mahadik and Shirke[2,3]. They studied the SVSSI $\bar{X}$ chart and SVSSI $T^{2}$ chart and have shown that both charts increase their statistical performances significantly by a numerical example. But the states whether the process is in control or out of control and the economical design are not considered.

In this paper, two statuses whether the process is in control or out of control are considered to define the new states of Markov Chain, and the economic performance on the SVSSI $T^{2}$ control chart is studied. Then a numerical example is provided to compare the performances of three control charts in terms of the adjusted average time to signal(AATS) and the expected lost(EL).

\section{Problem Description}

We assume that there are $p$ quality characteristics of product in process, and the distribution of the observations is follow $p$-variate normal with mean vector $\mu$ and known covariance matrix $\Sigma$. Let $X_{i j}$ be the $i$ th sample whose size is $n_{(i)}, i=1, \ldots, m, j=1, \ldots, n_{(i)}$, then the mean vector 
$\bar{X}_{i}=\frac{1}{n_{(i)}} \sum_{j=1}^{n_{(i)}} X_{i j}$. Let $\mu_{0}$ be the target mean vector. An occurrence of an assignable cause shifts $\mu$ from $\mu_{0}$ to $\mu_{1}$. Thus the control chart statistic is $T_{i}^{2}=n_{(i)}\left(\bar{X}_{i}-\mu_{0}\right)^{\prime} \Sigma^{-1}\left(\bar{X}_{i}-\mu_{0}\right)$. Note that when $\mu=\mu_{0}, T_{i}^{2}$ follows the central chi-square distribution with $p$ degrees of freedom, and when $\mu=\mu_{1}, T_{i}^{2}$ follows the non-central chi-square distribution with $p$ degrees of freedom and non-central parameter $\lambda_{i}=n_{(i)}\left(\mu_{1}-\mu_{0}\right)^{\prime} \Sigma^{-1}\left(\mu_{1}-\mu_{0}\right)=n_{(i)} d^{2}$, where $d$ is the Mahalanobis distance. When the assignable cause occurs, the process are required abnormal detection and maintenance, owing to the fact that it will not be automatically repaired.

For the SVSSI $T^{2}$ control chart, we select two sampling intervals $h_{1}$ and $h_{2}$ where $h_{1} \geq \boldsymbol{h}_{2}$, three sampling size $n_{1}, n_{2}$ and $n_{3}$ where $n_{1} \leq n_{2} \leq n_{3}$, two threshold limits $\omega_{1}$ and $\omega_{2}$ and static control limit $k$ where $0 \leq \omega_{1} \leq \omega_{2} \leq k$. So the control chart can be divided into four regions as follows. $I_{1}=\left[0, \omega_{1}\right], I_{2}=\left[\omega_{1}, \omega_{2}\right], I_{3}=\left[\omega_{2}, k\right]$ and $I_{4}=[k,+\infty)$. When $T_{i}^{2}$ falls in the in-control area, the values of the adaptive parameters of the chart for the next sampling are chosen by the following function.

$$
\left(n_{(i)}, h_{(i)}\right)=\left\{\begin{array}{l}
\left(n_{1}, h_{1}\right), T_{i-1}^{2} \in I_{1} \\
\left(n_{2}, h_{2}\right), T_{i-1}^{2} \in I_{2} \\
\left(n_{3}, h_{2}\right), T_{i-1}^{2} \in I_{3}
\end{array}\right.
$$

When $T_{i-1}^{2} \in I_{4}$, it is determined that the process is out of control.

\section{Economical Model}

\section{Statistical Indexes of SVSSI $T^{2}$ Control Chart}

There are three main indexes for measuring control chart performance, average time to signal(ATS), average number of sampling(ANOS) and average number of false alarm(ANF). The states build by Mahadik and Shirke are incomplete, and they did not consider the economic design. In this paper, based on the study, we consider two states weather it is controlled or out of control, and adding the state of false alarm to amend the state space. At the same time its economic design is designed and analyzed.

Let the controlled state be recorded as $I$ and the out of control state is denoted by $N$. Define eight states of Markov Chain, $\left\{I_{1}, I\right\},\left\{I_{2}, I\right\},\left\{I_{3}, I\right\},\left\{I_{4}, I\right\},\left\{I_{1}, N\right\},\left\{I_{2}, N\right\},\left\{I_{3}, N\right\},\left\{I_{4}, N\right\}$.

Assuming that the time at which the assignable cause occurs follows the exponential distribution of the parameter $\lambda$, then the probability of the occurrence is $1-e^{-\lambda h_{i}}, i=1,2$. When the process is in the state $\left\{I_{4}, I\right\}$, it is a false alarm, and when the process is in the state $\left\{I_{4}, N\right\}$, that is, absorption state. Then the transition probability matrix $P$ of the process is given as follows.

$$
P=\left(\begin{array}{cccccccc}
P_{11} & P_{12} & P_{13} & P_{14} & P_{15} & P_{16} & P_{17} & P_{18} \\
P_{21} & P_{22} & P_{23} & P_{24} & P_{25} & P_{26} & P_{27} & P_{28} \\
P_{31} & P_{32} & P_{33} & P_{34} & P_{35} & P_{36} & P_{37} & P_{38} \\
P_{41} & P_{42} & P_{43} & P_{44} & P_{45} & P_{46} & P_{47} & P_{48} \\
0 & 0 & 0 & 0 & P_{55} & P_{56} & P_{57} & P_{58} \\
0 & 0 & 0 & 0 & P_{65} & P_{66} & P_{67} & P_{68} \\
0 & 0 & 0 & 0 & P_{75} & P_{76} & P_{77} & P_{78} \\
0 & 0 & 0 & 0 & 0 & 0 & 0 & 1
\end{array}\right)
$$

where $P_{i j}$ represents the probability of transition from state i to state j, calculated as follows. 


$$
\begin{aligned}
& \text { Eq.1 } P_{i 1}=F\left(\omega_{1}, p, \eta_{0}\right) e^{-\lambda h_{i}}, i=1,2,3,4 \\
& \text { Eq.2 } P_{i 2}=\left[F\left(\omega_{2}, p, \eta_{0}\right)-F\left(\omega_{1}, p, \eta_{0}\right)\right] e^{-\lambda h_{i}}, i=1,2,3,4 \\
& \text { Eq.3 } P_{i 3}=\left[F\left(k, p, \eta_{0}\right)-F\left(\omega_{2}, p, \eta_{0}\right)\right] e^{-\lambda h_{i}}, i=1,2,3,4 \\
& \text { Eq.4 } P_{i 4}=\left[1-F\left(k, p, \eta_{0}\right)\right] e^{-\lambda h_{i}}, i=1,2,3,4 \\
& \text { Eq.5 } P_{i 5}=F\left(\omega_{1}, p, \eta_{i}\right)\left(1-e^{-\lambda h_{i}}\right), i=1,2,3,4 \\
& \text { Eq.6 } P_{i 6}=\left[F\left(\omega_{2}, p, \eta_{i}\right)-F\left(\omega_{1}, p, \eta_{i}\right)\right]\left(1-e^{-\lambda h_{i}}\right), i=1,2,3,4 \\
& \text { Eq.7 } P_{i 7}=\left[F\left(k, p, \eta_{i}\right)-F\left(\omega_{2}, p, \eta_{i}\right)\right]\left(1-e^{-\lambda h_{i}}\right), i=1,2,3,4 \\
& \text { Eq.8 } P_{i 8}=\left[1-F\left(k, p, \eta_{i}\right)\right]\left(1-e^{-\lambda h_{i}}\right), i=1,2,3,4 \\
& \text { Eq.9 } P_{i 5}=P\left\{0 \leq T^{2}<\omega_{1}\right\}=F\left(\omega_{1}, p, \eta_{i-4}\right), i=5,6,7 \\
& \text { Eq.10 } P_{i 6}=\left[F\left(\omega_{2}, p, \eta_{i-4}\right)-F\left(\omega_{1}, p, \eta_{i-4}\right)\right], i=5,6,7 \\
& \text { Eq.11 } P_{i 7}=\left[F\left(k, p, \eta_{i-4}\right)-F\left(\omega_{2}, p, \eta_{i-4}\right)\right], i=5,6,7
\end{aligned}
$$

Where $F(x, p, \eta)$ represents the cumulative probability distribution function of the non-central chi-square distribution with $p$ degrees of freedom and non-central parameter $\eta, \eta_{0}=0, \eta_{i}=n_{i} d^{2}$.

The values of AATS, ANOS and ANF can be calculated from matrix $P$.

$$
\begin{aligned}
& \text { Eq.13 AATS }=r^{\prime}(I-Q)^{-1} h-1 / \lambda \\
& \text { Eq.14 ANOS }=r^{\prime}(I-Q)^{-1} n \\
& \text { Eq.15 ANF }=r^{\prime}(I-Q)^{-1} f
\end{aligned}
$$

Where $\mathrm{Q}$ is the sub-matrix of $\mathrm{P}, h$ is the sample interval of each state, $r$ is the initial probability of the process, $f$ is the false alarm probability of each state, and $n$ is the sample size of each state. In order to avoid the initial problem of production and the easier calculation, choose that,

$h^{\prime}=\left(h_{1}, h_{2}, h_{2}, h_{2}, h_{1}, h_{2}, h_{2}\right), r^{\prime}=(0,0,1,0,0,0,0), f^{\prime}=(0,0,0,1,0,0,0), n^{\prime}=\left(n_{1}, n_{2}, n_{3}, n_{3}, n_{1}, n_{2}, n_{3}\right)$ where $r^{\prime}$ is the transpose of $r$.

\section{Economic Model of SVSSI $T^{2}$ Control Chart}

Each production cycle includes a controlled phase, a runaway phase, a detection phase, and a maintenance phase. In this paper, the Costa economic model is used[4].

$$
\text { Eq.16 } E L=V_{0}-\frac{E(C)}{E(T)}=V_{0}-\frac{V_{0}(1 / \lambda)+V_{1} * A A T S-C_{1} * A N F-A_{1}-C_{0} * A N O S}{A T C+T_{0} * A N F+T_{1}}
$$

Where $V_{0}$ represents the profit per hour when the process is in control, $V_{1}$ represents the hourly profit when out of control, $C_{0}$ is the cost of the individual sample, $C_{1}$ is the average cost of the search for the false alarm, $A_{1}$ is the expected cost of the error correction, $T_{0}$ is the average time of searching the false alarm, and $T_{1}$ indicates the average time for the error correction.

\section{Numerical Example}

\section{Comparisons of Charts}

In order to compare the performance of SVSSI, VSSI and VSI $T^{2}$ charts, the data from the study of Zhang Jing [5] and the parameters from Mahadik are used to verify, so that the average run time of the 
three control charts when it is in control is the same. The parameters of the above model are set as follows and the adaptive parameters of charts are shown in Table 1. And the values of AATS and EL of the three charts are shown in Table 2 and Table 3 for different offset. $p=2, \lambda=0.01, T_{1}=0.5 h, T_{2}=1.5 h, C_{0}=3.6 y$ yan, $C_{1}=115 y$ yan, $A_{1}=465$ yuan, $V_{0}=135 y$ yuan, $V_{1}=60$ yuan.

Table 1 The design parameters of control chart

\begin{tabular}{|l|l|l|l|}
\hline \multirow{2}{*}{ Control chart } & parameters & \multicolumn{3}{|l|}{$\boldsymbol{n}_{\mathbf{1}}, \boldsymbol{n}_{\mathbf{2}}, \boldsymbol{n}_{\mathbf{3}}$} \\
\cline { 2 - 4 } & $\boldsymbol{\omega}_{1}, \boldsymbol{\omega}_{2}$ & $\boldsymbol{h}_{\mathbf{1}}, \boldsymbol{h}_{\mathbf{2}}(\mathrm{h})$ & 3 \\
\hline $\mathrm{VSI}^{2}$ & 2.18 & $1.45,0.1$ & 1,7 \\
\hline $\mathrm{VSSI}^{2}$ & 2.18 & $1.45,0.1$ & $2,3,12$ \\
\hline $\mathrm{SVSSI}^{2}$ & $2.18,5.08$ & $1.45,0.1$ & $T^{2}$ \\
\hline
\end{tabular}

Table 2 The AATS values of control charts

\begin{tabular}{|l|l|l|l|}
\hline \multirow{2}{*}{$\mathrm{d}$} & \multicolumn{2}{|l|}{ AATS(h) } & \multicolumn{2}{|l|}{ SVSSI $^{2}$} \\
\cline { 2 - 4 } & VSI $T^{2}$ & VSSI $^{2}$ & 29.7849 \\
\hline 0.5 & 45.4475 & 31.6780 & 6.4630 \\
\hline 0.75 & 15.3918 & 7.6034 & 2.5715 \\
\hline 1 & 5.4994 & 3.0066 & 1.5850 \\
\hline 1.25 & 2.3137 & 1.9713 & 1.1708 \\
\hline 1.5 & 1.2695 & 1.5615 & 0.9628 \\
\hline 1.75 & 0.9144 & 1.3166 & 0.8507 \\
\hline 2 & 0.7869 & 1.1368 & \\
\hline
\end{tabular}

From the Table 2, it can be seen that when the shift in process is relatively small(i.e. $d \leq 1.5$ ), the AATS values of SVSSI $T^{2}$ control chart is smaller than that of the VSSI and VSI charts, which proves that the SVSSI $T^{2}$ control chart detect more sensitively for the small shift. And when the shift is large, the performance of SVSSI $T^{2}$ control chart is superior to the VSSI chart, and similar to the VSI chart.

Table 3 The EL values of control chart

\begin{tabular}{|l|l|l|l|}
\hline \multirow{2}{*}{$\mathrm{d}^{2}$} & EL[yuan] & \multicolumn{3}{|l|}{} \\
\cline { 2 - 4 } & VSI $T^{2}$ & VSSI $T^{2}$ & SVSSI $T^{2}$ \\
\hline 0.25 & 39.7237 & 35.7858 & 35.2726 \\
\hline 0.5 & 29.3012 & 25.2208 & 24.6703 \\
\hline 0.75 & 24.5294 & 22.0173 & 21.8504 \\
\hline 1 & 22.1624 & 20.8545 & 20.8707 \\
\hline 1.25 & 20.8899 & 20.3299 & 20.4106 \\
\hline 1.5 & 20.1572 & 20.0451 & 20.1466 \\
\hline 1.75 & 19.7107 & 19.8670 & 19.9756 \\
\hline 2 & 19.4254 & 19.7428 & 19.8559 \\
\hline
\end{tabular}

From the Table 3, it can be seen that when the shift in process is relatively small(i.e. $d^{2} \leq 0.75$ ), the EL values of SVSSI $T^{2}$ control chart is smaller than that of the VSSI and VSI charts, which means that it can save production costs. And when the shift is large, the EL values of the VSSI and SVSSI control charts are slightly larger than the VSI control chart.

In summary, compared to other two charts, the SVSSI $T^{2}$ control chart can speed up the detection of anomalies while reducing production costs, especially for the small shift.

\section{Optimal Parameter Selection}

In this paper, we assume that the process shift is $d^{2}=0.5$, the optimtool of algorithm toolbox in Matlab software is used to search the optimal solution of the economic design of the control chart so 
that the expected loss EL reaches the minimum value. Taking into account the actual situation and simpler calculation, set the economic model optimization conditions as follows:

$$
\begin{aligned}
& \min E L\left(n_{1}, n_{2}, n_{3}, h_{1}, h_{2}, w_{1}, w_{2}\right) \\
& \text { s.t.: } 0<n_{1}<n_{2}<n_{3}, n_{i} \text { is integer; } \\
& 2>\mathrm{h}_{1}>h_{2}>0 ; \\
& 0<\omega_{1}<\omega_{2}<k ;
\end{aligned}
$$

The optimal parameters of each control chart and its EL values are shown in Table 4.

Table 4 The optimal parameters of control charts

\begin{tabular}{|l|l|l|}
\hline \multirow{2}{*}{ Control chart } & \multicolumn{2}{|l|}{ Optimal parameters } \\
\cline { 2 - 3 } & $\left(\omega_{1}, \omega_{2}, h_{1}, h_{2}, n_{1}, n_{2}, n_{3}\right)$ & EL \\
\hline $\operatorname{VSI~}^{2}$ & $(1.651,1.999,0.746,5)$ & 28.34 \\
\hline${\text { VSSI } T^{2}}^{2}(1.652,1.997,0.128,1,5)$ & 25.77 \\
\hline SVSSI $T^{2}$ & $(3.027,4.604,1.999,1.449,1,8,19)$ & 21.52 \\
\hline
\end{tabular}

Thus, the SVSSI $T^{2}$ control chart is significantly better than the VSI and VSSI control charts. Therefore, in the production practice, according to the actual situation, SVSSI $T^{2}$ control chart can be chosen as an adaptive control chart to use for decreasing production costs.

\section{Summary}

In this paper, the SVSSI $T^{2}$ control chart is studied, and the new Markov Chain space is constructed by taking the in-control and out-of-control states into account. And compared it with the VSI and VSSI $T^{2}$ control charts at different shifts. The SVSSI $T^{2}$ control chart is superior to the other two control charts when the shift of mean value of the process is small. It is proved that the SVSSI $T^{2}$ control chart combines the advantages of the VSI and VSSI $T^{2}$ control charts.

Further, the sensitivity analysis can be carried out for each parameter of the cost function, and the actual influence relationship can be obtained, which can provide reference for enterprises. New optimization algorithm can be proposed. And the statistical performance can be combined to optimize the economic model.

\section{Acknowledgement}

This research was financially supported by the Humanity and Social Science Research and Planning Foundation of Ministry of Education of China (Grant No. 14YJA910004), National Social Science Foundation (Grant No. 16BTJ019), Jiangsu Provincial Natural Science Foundation of China (Grant No. BK20151481).

\section{References}

[1] Reynolds M R et al, $\bar{x}$ charts with variable sampling intervals, J. Technometrics, 30(2) (1988) 181-192.

[2] Mahadik S B, Shirke D T, A special variable sample size and sampling interval chart, J. Communications in statistics - theory and methods, 38 (2009) 1284-1299.

[3] Mahadik S B, Shirke D T, A special variable sample size and sampling interval Hotelling's $T^{2}$ chart, J. International journal of advanced manufacturing technology,53(2011) 379-384. 
[4] Maysa S , Costa, Economic-Statistical Control Chart Design: A Sensitivity Study, J. Brazilian Journal of Operations \& Production Management, 10(2) (2005) 25-38.

[5] Jing Z, The statistic and economic design of VSSI- $T^{2}$ control chart, D. Hunan university(2013). (In Chinese) 